\title{
PRESENTACIÓN Y ANÁLISIS DEL ESTADO DE RESULTADOS INTEGRALES EN EMPRESAS PERUANAS QUE ADOPTARON NORMAS IFRS
}

\author{
PRESENTATION AND ANALYSIS OF THE STATEMENT OF COMPREHENSIVE IN \\ PERUVIAN COMPANY ADOPT STANDARDS IFRS
}

\author{
Percy Vilchez Olivares* \\ Docente Asociado de la Facultad de Ciencias Contables \\ Universidad Nacional Mayor de San Marcos-UNMSM / Lima-Perú
}

[Recepción: Mayo de 2013/ Conformidad: Junio de 2013]

\section{RESUMEN}

Con el propósito de brindar información útil a los diversos usuarios de la información financiera, tales como los inversionistas, acreedores, prestamistas, sobre los resultados obtenidos en un período determinado, diversos organismos contables emisores de normas de alcance nacional e internacional en las dos últimas décadas han venido implementando como parte de su normatividad la obligación de presentar información financiera de los resultados integrales que ha obtenido una entidad. Adicionalmente, es importante señalar que existen diversos estudios e investigaciones contables que sustentan la utilidad de contar con esta información como complemento de la información financiera preparada para propósitos generales.

En el presente trabajo de investigación, se analizara las características de los resultados integrales, sus componentes, y la normatividad contable de las NIIF sobre la presentación del estado de los resultados integrales. Asimismo, busca identificar que empresas de las que cotizan en la Bolsa de Valores de Lima presentan resultados del período con montos diferentes a los resultados Integrales en el período 2011, y sobre esa información analizar los tipos de variaciones que se presentan entre dichos resultados, la frecuencia de los componentes de los ingresos y gastos reconocidos en el patrimonio determinantes del resultado integral, y la significación monetaria de las variaciones entre el resultado del período y el resultado integral.

Palabras claves: normas internacionales de información financiera, resultados integrales, impuesto a las ganancias diferidas, mercado bursátil, usuarios de la información financiera

\begin{abstract}
In order to provide useful information to the various users of financial information, such as investors, creditors, lenders, on the results obtained in a given period, various accounting bodies rules issuers of national and international scope in the last two decades have been implemented as part of its provisions required to submit financial information obtained income that have an entity. Additionally, it is important show there are several accounting studies and research that support the usefulness of this information in addition to prepared for general purposes.

In the present work of investigation, it analyzed the characteristics of the results comprehensive income, its components, and the IFRS accounting standards on the presentation of the statement of comprehensive income. It also seeks to identify companies that are listed on the Lima Stock Exchange for the period presented results with different amounts Comprehensive results for the period 2011, and on that information to analyze the types of variations that occur between these results, the frequency components of income and expense recognized in equity determinants of comprehensive income, and monetary significance of changes between profit or loss and comprehensive income.

Keywords: international financial reporting standards, comprehensive income, deferred income taxes, stock market, financial information users
\end{abstract}

* Magister en Ciencias Contables y Financieras - Universidad Diego Portales (Chile). E-mail: pvilchezcpa@gmail.com 


\section{INTRODUCCIÓN}

En nuestro país a partir del período 2011 las empresas que cotizan en la Bolsa de Valores de Lima tienen la obligación de reportar su información financiera bajo las Normas Internacionales de Información Financiera (IFRS por siglas en inglés), con lo cual se logra una mayor calidad y cantidad de revelaciones en la información financiera, lo anterior significa la aplicación irrestricta de todas las normas emitidas por la Junta de Normas de Contabilidad Financiera (organismo emisor de las Normas Internacionales de Información Financiera NIIF).

Como parte del actual marco normativo de las NIIF, se encuentra vigente la Norma Internacional de Contabilidad NIC 1 Presentación de estados financieros, dicha norma incluye la información principal sobre la regulación contable del estado de resultados integrales, el mismo que puede analizarse con normas particulares que tratan sobre los mismos.

Uno de los hechos más importantes en la adopción de estándares contables internacionales en el mundo es la decisión que tuvo la Unión Europea en el año 2002 en la que obliga a todas las empresas que participan en mercados bursátiles de preparar información financiera bajo el modelo contable de las Normas Internacionales de Información Financiera a partir del año 2005. Este hecho implicó que alrededor de 7,000 compañías de Europa presenten información bajo estos estándares contables internacionales Considero que es uno de los hechos más importantes hacia el uso generalizado de estos estándares y con el cual se inicia un proceso acelerado de adopción de las Normas internacionales de Información Financiera en diversos países del mundo. Hoy en día globalmente más de 100 países utilizan total o parcialmente estos estándares contables internacionales.

Las Normas Internacionales de Información Financiera (IFRS, por sus siglas en inglés), son emitidas por la Junta de Normas Internacionales de Contabilidad (IASB por sus siglas en inglés), son estándares internacionales que tienen como objetivo aumentar la transparencia y utilidad de la información en los mercados capitales globales, contar con procesos contables bajo un modelo contable único, poseer facilidad de revisión de información financiera por organismos reguladores.

Por otro lado, en el Perú las empresas que tenían cotización en el mercado bursátil, reportaron información financiera de acuerdo a lo dispuesto en el artículo 223 de la Ley General de Sociedades de acuerdo a los denominados principios de contabilidad generalmente aceptados en el Perú hasta el año 2010, los cuales implicaban la sustentación en diversas normas IFRS, aplicación de diversas normativas contables nacionales, regulaciones contables legales sectoriales, prácticas contables y eventualmente regulaciones tributarias, entre otros. Pero, considerando que el párrafo 14 de la NIC 1 Presentación de Estados Financieros indica que la administración de la empresa solo podrá afirmar en nota a sus estados financieros que estos han sido preparados de acuerdo con Normas Internacionales de Información Financiera si ha cumplido con todos los requerimientos del conjunto de normas que comprenden las Normas Internacionales de Información Financiera; por lo tanto, podemos llegar a la conclusión que la información financiera preparada de acuerdo a los Principios de Contabilidad Generalmente Aceptados en el Perú son distintos de los que resultarían de la aplicación integral de las Normas Internacionales de Información Financiera.

En ese sentido, para efectos de llevar un proceso de convergencia de Principios Contables Generalmente Aceptados (PCGA) locales hacia las Normas Internacionales de Información Financiera, la Comisión Nacional Supervisora de Empresas y Valores CONASEV (hoy Superintendencia del Mercado de Valores) el 10 de octubre del 2010 emitió la Resolución $\mathrm{N}^{\circ}$ 102-2010-EF/94.01.1 mediante la cual dispone que las empresas bajo su supervisión y de observancia para las demás empresas, deben preparar sus estados financieros con observancia plena de las Normas Internacionales de Información Financiera a partir de los estados financieros por el año que termina el 31 de diciembre del 2011. Para ello se debe observar la "NIIF 1 Adopción por Primera Vez de las 
Normas Internacionales de Información Financiera”.

En el año 2003 el IASB organismo emisor de las Normas Internacionales de Información Financiera publicó la NIIF 1 Adopción por Primera Vez de las Normas Internacionales de Información Financiera, la cual tiene como objetivo asegurar que los primeros estados financieros NIIF de la entidad contengan información de alta calidad que sea tranparente para los usuarios y comparable durante todos los períodos que se presenten, proporcionen un punto de partida confiable para la contabilidad según las Normas Internacionales de Información Financiera y que pueda ser generada a un costo que no exceda los beneficios de los usuarios.

Por lo expuesto, las empresas supervisadas por la Superintendencia del Mercado de Valores cumplieron con presentar información financiera anual por el período 2011 por primera vez bajo Normas Internacionales de Información Financiera, con lo cual se inicia en nuestro país un proceso de adaptación de la información financiera al nuevo modelo normativo que constituyen las Normas Internacionales de Información Financiera. En esa línea el presente trabajo de investigación pretende analizar la relevancia y representación fiel de la información financiera suministrada por el Estado de Resultados Integrales en aplicación de la NIC 1 Presentación de Estados Financieros.

\section{Marco de Referencia}

\subsection{Importancia de la Información del Resulta- do Integral para los Usuarios de la Información Financiera}

En la literatura contable americana existen diversos autores que han planteado la utilidad en la información que presentan los resultados integrales, en el sentido que al informar los resultados integrales se aporta información relevante sobre los cambios en el patrimonio no relacionados con transacciones con los accionistas. Algunos autores de la escuela clásica normativa deductiva que cabe señalar son MacNeal, K. en 1939 (Truth un Accounting, Univeristy of Pennsylvania), Edwards, E. y Bell, P. en 1961 (The
Theory and Measurement of Business Income, University of California), y Sprouse, R. and Moonitz, M. en 1962 (A Tentative Set of Broad Accounting Principles for Business Enterprises, American Institute of Certified Public Accountants, New York).

Respecto a investigaciones empíricas efectuadas en Estados Unidos asociadas al impacto en los usuarios de los formatos para la presentación del resultado integral, podemos señalar la efectuada por Hirst y Hopkins en 1998 para evaluar la incidencia de los formatos alternativos de presentación del resultado integral según el SFAS 130 del FASB en la detección de prácticas earnings management, teniendo como muestra 96 analistas financieros del CFA Institute, en la que se indica si el resultado global se revela de forma nítida en el estado de resultados integrales, y no escondido en el estado de cambios en el patrimonio neto, se facilita la detección de prácticas de earnings management, hasta el punto que los analistas financieros asignan un precio similar a las acciones de empresas que llevan a cabo dichas prácticas y las que no siguen las mismas. Asimismo, un estudio similar fue efectuado por Hunton et al. en 2006 teniendo como muestra a 62 profesionales de la dirección financiera y CEO con similares conclusiones.

Por su parte, Sousa Francisco de España en el 2008 realiza una investigación empírica para estudiar el impacto del resultado integral frente al resultado neto, revelados ambos según la NIC 1 del IASB, tanto para el conjunto de la muestra como particularizado por sectores, teniendo como muestra a 92 empresas cotizadas españolas en la Bolsa de Madrid (2004-2007), en la que se indica que el resultado global frente al resultado neto presenta diferencias estadísticamente significativas para el conjunto de la muestra en los cuatro años estudiados. Sin embargo, apenas existen diferencias significativas entre los distintos sectores de actividad económica, con lo que nos encontramos ante un fenómeno, que en líneas generales afecta a cotizadas en todos los sectores.

De las investigaciones referidas e incluidas en el artículo Fundamentos Conceptuales del Resultado Global, preparado por Sousa Francisco en el 2009 podemos precisar que es relevante la información que 
brinda los resultados integrales para los inversionistas, prestamistas y otros acreedores como usuarios de la información financiera en el proceso de toma de decisiones de inversión, financiación y operación.

Asimismo, diversos organismos reguladores contables a nivel internacional desde la década de los noventa del siglo XX, han incorporado a su normatividad contable los resultados integrales, por ejemplo el organismo emisor FASB de Estados Unidos emitió la norma SFAS 130 "Reporting Comprehensive Income” en el año 1997, el organismo emisor ASB de Inglaterra emitió la norma FRS 3 "Reporting Financial Performance" en el año 1999, y el organismo emisor IASB que tiene alcance internacional emitió la Modificación de la NIC 1 "Presentación de Estados Financieros" en el año 2007, asimismo, recientemente el organismo emisor de normas CINIF de México ha emitido el documento B-3 "Estado de Resultado Integral" en el año 2011.

Por lo expuesto, esta nueva concepción de revelación de los resultados integrales, si bien es cierto tiene fundamentos vinculados al paradigma de la utilidad de la información y coincidencia entre los diversos modelos normativos mundiales, también debo señalar que abandona el principio contable de realización, debido a que lo sustituye por el denominado principio de valor, donde no importa si los resultados estén realizados o no, sino que lo relevante para reconocer los ingresos o gastos es que los activos o pasivos hayan cambiado de valor, y evidentemente puedan medirse confiablemente. Esta aseveración es coincidente con el nuevo Marco Conceptual de Preparación de Información Financiera emitido en el 2010 por el IASB.

\subsection{Presentación del Estado de Resultado Integral según la Norma Internacional de Contabilidad Número 1 (NIC 1 Presentación de Estados Fi- nancieros)}

El Consejo de Normas Internacionales de Contabilidad (IASB) en el 2001 adoptó la NIC 1 Presentación de Estados Financieros, que había sido originalmente emitida por el Comité de Normas internacionales de Contabilidad en 1997.
En el 2003 el IASB emitió una NIC 1 revisada como parte de una agenda inicial de proyectos técnicos. Asimismo, el IASB emitió en el 2007 una NIC 1 modificada, que incluía una modificación de la presentación de los cambios en el patrimonio que proceden de los propietarios y del resultado integral y un cambio en la terminología de las denominaciones de los estados financieros. Finalmente en el 2011 el IASB modificó la NIC 1 para mejorar la forma de presentación de las partidas de otro resultado integral.

El objetivo de la NIC 1 es establecer las bases para la presentación de los estados financieros de propósito general, para asegurar que los mismos sean comparables, tanto con los estados financieros de la misma entidad correspondientes a periodos anteriores, como con los de otras entidades. Esta Norma establece requerimientos generales para la presentación de los estados financieros, guías para determinar su estructura y requisitos mínimos sobre su contenido.

La NIC 1 afecta la presentación de los cambios en el patrimonio que proceden de los propietarios y en el resultado integral. Lo anterior, no significa que se modifiquen los criterios de reconocimiento o medición de las partidas patrimoniales.

La norma requiere que se presente en el estado de cambios en el patrimonio todos los cambios o transacciones que proceden de los propietarios (por ejemplo, aporte de capital, declaración de dividendos), de forma separada de los cambios distintos relacionados con los propietarios en el patrimonio (por ejemplo, superávit por revaluación, ganancia por activos financieros disponibles para la venta); asimismo, se indica que todos los cambios o transacciones en el patrimonio que no proceden de los propietarios se requiere que se presenten en un estado de resultado integral o en dos estados (un estado de resultado separado y un estado de resultado integral) los componentes del resultado integral no está permitido que se presenten en el estado de cambios en el patrimonio. El propósito es proporcionar mayor información mediante la agregación de partidas con características comunes y separando partidas con características diferentes. 
Se requiere que se revele los resultados e impuestos a las ganancias vinculados con cada componente de otro resultado integral. Lo anterior se explica porque los componentes a menudo tienen tasas fiscales diferentes de las aplicadas al resultado. Asimismo, requiere que se revele los ajustes por reclasificación relacionados con los componentes de otro resultado integral. Los ajustes por reclasificación son importes reclasificados en el resultado del período corriente que fueron reconocidos en otro resultado integral en períodos anteriores.

El resultado integral se presenta en los estados financieros que emita una entidad al final del período del que se informa.

\section{Anexo 1 Estructura de Componentes del Resultado Integral}

\begin{tabular}{|c|c|}
\hline Componentes & Referencia a la NIC \\
\hline \multicolumn{2}{|l|}{$\begin{array}{l}\text { Ganancia (Pérdida) Neta del Ejercicio } \\
\text { Componentes de Otro Resultado Intearal }\end{array}$} \\
\hline Componentes de Otro Resultado Integral & \\
\hline Cobertura de flujo de efectivo & NIC 39 y NIIF 9 \\
\hline Cobertura de inversión neta en negocios en el extranjero & CINIIF 16 \\
\hline $\begin{array}{l}\text { Variaciones de inversiones en instrumentos de patrimonio al valor } \\
\text { razonable }\end{array}$ & NIC 39 y NIIF 9 \\
\hline Diferencia de cambio por conversión en operaciones en el extranjero & NIC 21 \\
\hline $\begin{array}{l}\text { Ganancia (Pérdida) en activos o pasivos financieros disponibles para } \\
\text { la venta }\end{array}$ & NIC 39 y NIIF 9 \\
\hline Superávit de revaluación & NIC 16 y NIC 38 \\
\hline Ganancia (Pérdida) actuariales en plan de beneficios definidos & NIC 19 \\
\hline $\begin{array}{l}\text { Cambios en el valor razonable de pasivos financieros atribuibles a } \\
\text { cambios en el riesgo de crédito del pasivo }\end{array}$ & NIC 39 y NIIF 9 \\
\hline $\begin{array}{l}\text { Impuesto a las ganancias relacionados con componentes de otro resultado } \\
\text { integral }\end{array}$ & NIC 12 \\
\hline Otro resultado integral & \\
\hline Resultado Integral del Ejercicio Net & \\
\hline
\end{tabular}

Fuente: Superintendencia del Mercado de Valores

El Estado del Resultado Integral incluirá como mínimo los siguientes rubros:

$\mathrm{Si}$ bien es cierto, el formato incluye las últimas modificaciones de las Normas Internacionales de Información Financiera, es necesario que se revise la eventual creación de cuentas en el Plan Contable General Empresarial para los componentes integrales que no tienen cuenta contable a nivel del elemento 5 (cinco) Patrimonio Neto.

\section{Análisis de la Presentación del Resultado} Integral para Empresas Cotizadas en Perú

Para analizar la información financiera presentada a la actual Superintendencia del Mercado de Valores en el período 2011 se tomó un grupo de empresas individuales cotizadas en el mercado bursátil, que obligatoriamente presentaron por primera vez sus estados financieros bajo las Normas IFRS. Debo precisar, que algunas empresas que a pesar de estar cotizadas en el mercado bursátil no presentaron estados financieros bajo IFRS por estar regulada contablemente por otros organismos reguladores o porque solicitaron postergación para efectuar la adopción en el año 2012.

Para efectos de analizar la información de las empresas se definieron como componentes principales al resultado del período, los componentes del integral y los resultados integrales en el período 2011. Asimismo, se consideró el formato vigente utilizado por la Superintendencia de Mercado de Valores en el período 2011.

El presente análisis tiene por objetivos principales identificar las principales variaciones entre el resultado del período y el resultado integral, es decir las diversas posiciones de aumento o disminución 
que se pueden presentar entre ambas variables, los principales componentes de ingresos y gastos reconocidos en patrimonio que forman parte de los resultados integrales, y la tasa de aumento o disminución promedio respecto a los importes obtenidos por las empresas que han tenido cambios entre el resultado del período y resultado integral.

\subsection{Descripción de la Muestra}

De las 289 empresas cotizadas que presentaron estados financieros en el año 2011, se han considerado 54 empresas (18.69\%) que han presentado información de cambios entre el resultado del período y el resultado integral (Ver Anexo 2).

Anexo 2 Determinación de la Muestra Utilizada

\begin{tabular}{|l|c|c|}
\hline Grupo de Empresas & $\begin{array}{c}\text { Número de } \\
\text { empresas }\end{array}$ & $\begin{array}{c}\text { \% de } \\
\text { Participación }\end{array}$ \\
\hline $\begin{array}{l}\text { Empresas registradas para presentar estados financieros } \\
\text { individuales a la Bolsa de Valores de Lima }\end{array}$ & 289 & $100.00 \%$ \\
\hline $\begin{array}{l}\text { Empresas cuyo resultado del período es igual al resultado } \\
\text { integral }\end{array}$ & -118 & $-40.83 \%$ \\
\hline $\begin{array}{l}\text { Empresas que no aplican IFRS por corresponder a Bancos, } \\
\text { Sias. de Seguros, AFP; y otras instituciones financieras }\end{array}$ & -80 & $-27.68 \%$ \\
\hline $\begin{array}{l}\text { Empresas que reportaron información pero que no } \\
\text { complementaron el anexo del estado de resultado integral }\end{array}$ & -10 & $-3.46 \%$ \\
\hline $\begin{array}{l}\text { Empresas que la información financiera no está disponible } \\
\text { (generalmente fondos de inversión) }\end{array}$ & -24 & $-8.30 \%$ \\
\hline $\begin{array}{l}\text { Empresas que llenaron información con inconsistencia o no } \\
\text { presentaron información de resultados. }\end{array}$ & -3 & $-.04 \%$ \\
\hline$=$ Total Muestra Seleccionada & $\mathbf{5 4}$ & $\mathbf{1 8 . 6 9 \%}$ \\
\hline
\end{tabular}

Fuente: Elaboración Propia

\subsection{Determinación de las Variaciones entre el} Resultado del Período y el Resultado Integral

En las 54 empresas analizadas se han presentando cambios en las diversas relaciones entre el Resultado del Período (RP) y el Resultado Integral (RI), por lo que tomando de referencia el trabajo desarrollado por Jara y Abarca (2009) se confeccionó el Anexo 3 donde se muestra las seis alternativas de clasificación para las empresas que presentaron Resultados del Período con monto diferente al Resultado Integral, de tal forma conocer la concentración que se ha presentado en cada campo y el tipo de variación obtenida en las 54 empresas analizadas en el período 2011.

Anexo 3 Determinación de Cambios por Tipo y Efecto en la variación del Resultado del Período (RP) al Resultado Integral (RI)

\begin{tabular}{|c|c|c|c|c|}
\hline \multirow{2}{*}{\multicolumn{2}{|c|}{$\begin{array}{l}\text { Tipo } \\
\text { Variación }\end{array}$}} & \multicolumn{2}{|c|}{ Efecto Variación } & \multirow{2}{*}{$\begin{array}{c}\text { Total } \\
\text { Empresas }\end{array}$} \\
\hline & & Aumento & Disminución & \\
\hline$+\mathrm{RP}$ & $\mathrm{a}+\mathrm{RI}$ & $\begin{array}{c}35 \\
\text { Grupo A }\end{array}$ & $\begin{array}{c}16 \\
\text { Grupo "B" }\end{array}$ & 51 \\
\hline + RP & a $\quad-$ RI & No Aplicable & $\stackrel{2}{\text { Grupo C }}$ & 2 \\
\hline - RP & $a-R I$ & $\begin{array}{c}0 \\
\text { Grupo D }\end{array}$ & $\begin{array}{c}0 \\
\text { Grupo "E" }\end{array}$ & 0 \\
\hline - RP & $\mathrm{a}+\mathrm{RI}$ & $\begin{array}{c}1 \\
\text { Grupo F }\end{array}$ & No Aplicable & 1 \\
\hline Total & & 36 & 18 & 54 \\
\hline
\end{tabular}
resultado de pérdida.

Fuente: Elaboración Propia

92/ QVIPUKAMAVOC | Vol. 21(39) 2013 
Tal como se puede apreciar en el Anexo 3 la mayor concentración se presentó en el Grupo A que generó un aumento (positivo) en 35 empresas (64.81\%) del total de empresas analizadas, y lo inverso que significa una disminución (negativo) en 16 empresas (29.63\%) del total de empresas analizadas. Asimismo, el Grupo F que corresponde a la posición más positiva de las concentraciones se presentó en 1 empresa (0.02\%) del total de empresas analizadas, y el Grupo C que corresponde a la posición más negativa se presento solo en dos empresas $(0.04 \%)$ del total; por lo expuesto, la variación de aumento ha sido de 36 empresas es decir un $66.67 \%$ del total y una variación de disminución de 18 empresas es decir un $33.33 \%$ del total, con lo cual es mayor la proporción de empresas, peruanas analizadas que han tenido cambios positivos entre el resultado del período y el resultado integral obtenido al final del período 2011.

Las 35 empresas del Grupo A donde han tenido una variación de aumento en la relación $+\mathrm{RP} a+\mathrm{RI}$ que se presentan en orden de presentación en la página web de la SMV son las siguientes:

\begin{tabular}{|l|}
\hline 1. Comercial del Acero S.A. \\
\hline 2. Negocios e Inmuebles S.A. \\
\hline 3. Internacional de Títulos Sociedad Titulizadora S.A. \\
\hline 4. Agro Industrial Paramonga S.A.A. \\
\hline 6. Quuthern Peru Copper Corporation, Sucursal del Perú \\
\hline 7. Agropindustrias AlB S.A. \\
\hline 8. Reactivos Nacionales S.A. \\
\hline 9. Kallpa Generación S.A. \\
\hline 10. Castrovirreyna Compañía Minera S.A. \\
\hline 11. Supermercados Peruanos S.A. \\
\hline 12. Transacciones Financieras S.A. \\
\hline 13. Inversiones La Rioja S.A. \\
\hline 14. Graña y Montero S.A.A. \\
\hline 15. Inversiones Centenario S.A.A. \\
\hline 16. Edegel S.A.A. \\
\hline 17. Southern Cone Power Perú S.A. \\
\hline 18. Luz del Sur S.A.A. \\
\hline 19. Edelnor S.A.A. \\
\hline 20. Chinango S.A.C. \\
\hline 21. Alicorp S.A.A. \\
\hline 22. Transportadora del Gas del Perú S.A. \\
\hline 23. Ferreyros S.A.A. \\
\hline 24. Fábrica Nacional de Acumuladores Etna S.A. \\
\hline 25. Empresa Siderúrgica del Perú S.A.A. \\
\hline 26. EAFC Maquisistema S.A. \\
\hline 27. Minera Andina de Exploraciones S.A.A. \\
\hline 28. Telefónica Móviles S.A. \\
\hline 29. Futura Consorcio Inmobiliario S.A. \\
\hline 30. Volcán Compañía Minera S.A.A. \\
\hline 31. Sociedad Minera El Brocal S.A.A. \\
\hline 32. Consorcio Industrial de Arequipa S.A. \\
\hline 33. Laive S.A. \\
\hline 34. ENESUR S.A. \\
\hline 35. Cervecería San Juan S.A. \\
\hline
\end{tabular}


Las 16 empresas del Grupo B donde han tenido una variación de disminución en la relación $+\mathrm{RP} a+\mathrm{RI}$ que se presentan en orden de presentación en la página web de la SMV son las siguientes:

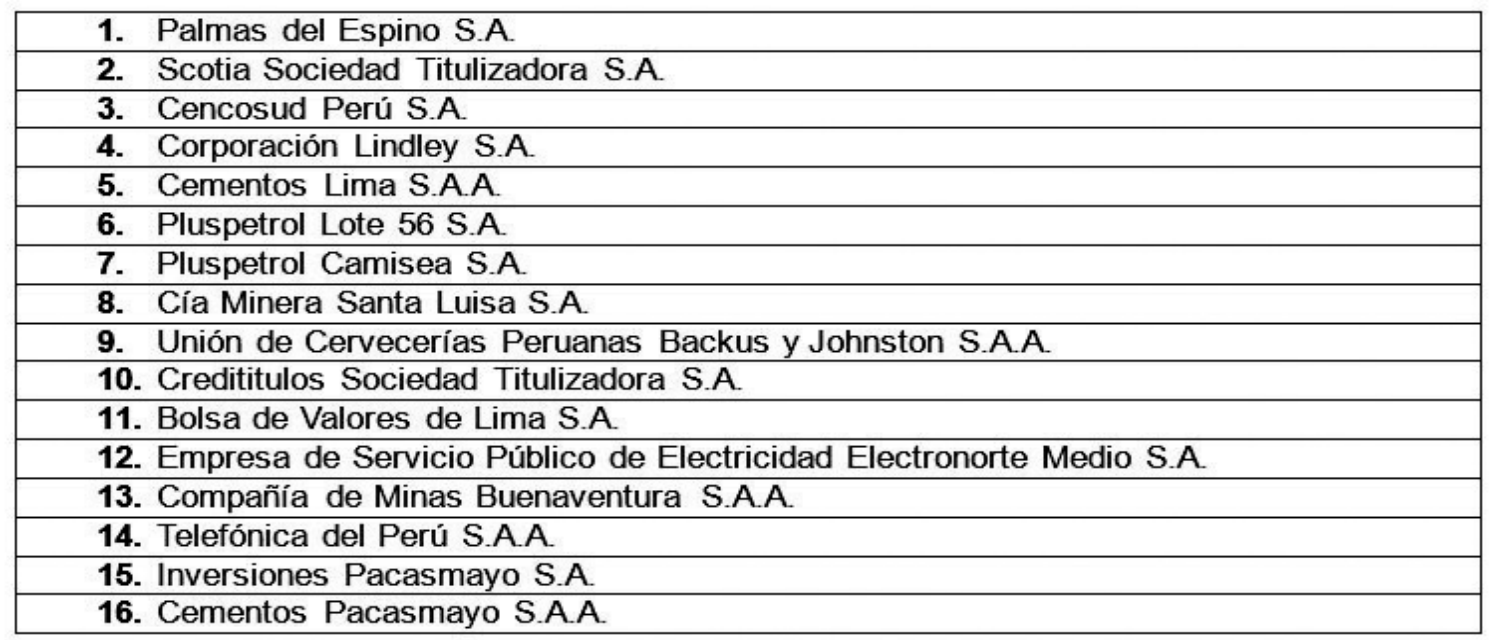

Las 2 empresas del Grupo $C$ donde han tenido una variación de disminución en la relación $+\mathrm{RP}$ a -RI que se presentan en orden de presentación en la página web de la SMV son las siguientes:

\section{Corporación Cervesur S.A.A}

2. Perú LNG S.R.L.

Finalmente 1 empresa que se concentra en el Grupo $F$ ha tenido una variación de aumento en la relación $-\mathrm{RP} \mathrm{a}+\mathrm{RI}$ y que es la empresa Lima Caucho S.A.

\subsection{Determinación de los Componentes del}

\section{Resultado Integral}

Para efectos de determinar los componentes de los ingresos y gastos que se reconocen en el patrimonio de las empresas que presentan cambios entre el resultado del período y el resultado integral, se analizó los componentes de las 54 empresas analizadas, pero bajo el formato que se utilizó por la SMV en la información financiera del período 2011.

El proceso que se realizó fue obtener los reportes financieros, tabular los registros que se presentan (en algunas empresas se presentan dos o más tipos de componentes de resultados integrales por empresa), validar la consistencia de las cifras, para obtener las frecuencias acumuladas para las 54 empresas. Las cifras obtenidas se presentan en el Anexo 4 Determinación de los componentes que configuran el resultado integral.

\section{Anexo 4 Determinación de Componentes Determinantes en la Configuración del Resultado Integral}

\begin{tabular}{|l|c|c|}
\hline \multicolumn{1}{|c|}{ Componentes de Otro Resultado Integral } & $\begin{array}{l}\mathrm{N}^{\circ} \text { de } \\
\text { eventos }\end{array}$ & $\begin{array}{l}\text { \% del } \\
\text { Total }\end{array}$ \\
\hline Ganancia (pérdida) por activos financieros disponibles para la venta & 15 & 23.44 \\
\hline Variación neta por cobertura de flujos de efectivo & 21 & 32.81 \\
\hline Ganancia (pérdida) de inversiones en instrumentos de patrimonio & 4 & 6.25 \\
\hline Diferencia de cambio por conversión en operaciones en el extranjero & 0 & 0.00 \\
\hline Participación por otro resultado integral de asociadas & 2 & 3.12 \\
\hline Superávit de revaluación & 11 & 17.19 \\
\hline Otros componentes del resultado integral & 11 & 17.19 \\
\hline Total & 64 & 100.00 \\
\hline
\end{tabular}


Tal como se puede apreciar en el Anexo 4, las 54 empresas analizadas han reportado 64 eventos en los resultados integrales, lo cual significa que por cada empresa se han realizado en promedio 1.18 eventos, lo cual significa una relación de una empresa un evento.

Asimismo, el componente variación neta de cobertura de flujos de efectivo ha presentado 21 eventos de los 64 eventos alcanzando la primera preferencia con $32.81 \%$ y el componente ganancia (pérdida) por activos financieros disponibles para la venta ha presentado 15 eventos de los 64 eventos alcanzados obteniendo la segunda preferencia con $23.44 \%$.

\subsection{Determinación de la Significancia Monetaria de los Cambios entre el Resultado del Período y Resultado Integral}

En la determinación de la significancia monetaria se ha agrupado los valores del resultado del período (utilidad o pérdida) obtenido y se ha comparado con la agrupación de los valores del resultado integral (utilidad o pérdida) de las 54 empresas analizadas, de tal forma que pueda obtener la variación monetaria del cambio y la tasa de aumento o disminución entre el resultado del período y el resultado integral.

Para efectos de poder analizar los cambios, el Anexo 5 presenta el monto de los resultados del período obtenido por grupo de empresas que se determinaron en el anexo 3 de la presente investigación.

\section{Anexo 5 Determinación de Significancia Monetaria de las Variaciones entre el Resultado del Período y el Resultado Integral (Expresado en miles de soles)}

\begin{tabular}{|c|c|c|c|}
\hline Grupos & Resultado Período & Resultado Integral & Variación \\
\hline A & $4,398,363$ & $4,665,131$ & $+266,768$ \\
\hline B & $2,623,750$ & $2,532,194$ & 91,556 \\
\hline C & 38,482 & $-78,026$ & - \\
\hline F & $-1,926$ & 57,694 & $+116,508$ \\
\hline Total & $7,058,669$ & $7,176,933$ & 59,620 \\
\hline
\end{tabular}

Por los resultados presentados en el Anexo 5 que muestra los resultados de las 54 empresas analizadas se ha obtenido que el resultado consolidado del período 2011 ascendió en miles de soles a S/.7,058,669 y el resultado integral consolidado por el mismo período ascendió en miles de soles a S/. 7,176,933, lo cual significó un aumento en miles de soles de S/.118,324, dicho monto significó un aumento relativo del resultado integral respecto al resultado del período en $1.68 \%$, dicha cifra no es significativa respecto al conjunto de todas las empresas, pero también debo señalar que algunas empresas han tenido cambios importantes entre los resultados del período y los resultados integrales, que individualmente tienen tasas superiores al 20\%, lo cual amerita la importancia de revelar los resultados integrales como estado financiero.

Las empresas del Grupo A que han tenido cambios importantes positivos superiores al $20 \%$ por ejemplo corresponde a las empresas Agroindustrias AIB S.A. con 27\%, Reactivos Nacionales S.A. con
35\%, Inversiones la Rioja S.A. con 97\%, Southern Cone Power Perú S.A. con 28\%, Ferreyros S.A.A. con $21 \%$, EAFC Maquisistema S.A. con 63\%, Sociedad Minera Brocal S.A.A. con 22\%, Consorcio Industrial de Arequipa S.A. con $289 \%$ y Laive S.A. con $97 \%$

Las empresas del Grupo B que han tenido cambios importantes menores superiores al $20 \%$ por ejemplo corresponde a las empresas Cencosud Perú S.A. con 98\%, Corporación Lindley S.A. con 52\%, e Inversiones Pacasmayo S.A. con 57\%.

\section{CONCLUSIONES}

1. El modelo normativo contable que se aplica para las empresas que cotizan en la Bolsa de Valores de Lima a partir del período 2011 es el modelo contable de las normas emitidas por la Junta de Normas de Contabilidad Financiera (IASB, por sus siglas en inglés) denominadas Normas Internacionales de Información Financiera, las mismas que se aplican en forma integral e 
irrestricta, por lo que es imprescindible tener un conocimiento adecuado de las revelaciones exigidas por estas normas, y muy en especial de los cambios en las revelaciones de los resultados del período.

2. Las empresas que tienen hechos patrimoniales que signifiquen un aumento en el patrimonio diferente de las transacciones realizadas entre la entidad y los accionistas, aparte de revelar la información en el estado de cambios en el patrimonio ahora en forma global), debe adicionalmente presentar un detalle de dichos cambios en un estado de resultados integrales bajo lo prescrito por la NIC 1 Presentación de estados financieros.

3. Del análisis realizado a las 289 empresas que presentaron información financiera individual del período 2011 a la Superintendencia del Mercado de Valores, se pudo confirmar que $54 \mathrm{em}$ presas (18.69\% del total) presentaron información financiera en la que el resultado del período difiere del resultado integral.

4. De la muestra obtenida que alcanza a 54 empresas, y producto de la comparación entre el resultado del período y el resultado integral (ambos con utilidades), se obtuvo que 35 empresas que tuvieron un aumento de los resultados, $16 \mathrm{em}$ presas una disminución de los resultados. Otra posición de comparación efectuada entre el resultado del período (utilidad) y el resultado integra (pérdida), se obtuvo que dos empresas tuvieron una disminución de sus resultados por el efecto de los integrales y la comparación entre el resultado del período (pérdida) y el resultado integral (utilidad) fue de una empresa que tuvo una posición positiva por efecto de los integrales.

5. Existen diversos componentes patrimoniales del resultado integral, siendo la variación neta por cobertura de flujo de efectivo y la ganancia (pérdida) por activos financieros disponibles para la venta, los componentes más frecuentes que se presentan en la información financiera reportada en el 2011 por las empresas analizadas.
6. La significancia monetaria en el año 2011 de las 54 empresas que cotizan en la Bolsa de Valores de Lima que presentan variaciones entre el resultado del período (monto total en miles de soles por $S / 7,058,669)$ y el resultado integral ( monto total en miles de soles por $S / 7,176,933$ ) asciende a la suma de S/ 118,324, dicho monto significó un aumento del resultado integral respecto al resultado del período en $1.68 \%$, dicha cifra no posee significancia monetaria por el conjunto de las empresas: pero cuando se analizan individualmente, algunas de estas pueden presentar variaciones superiores al $20 \%$, con lo cual se amerita la importancia de revelar los resultados integrales dentro del conjunto de información financiera.

\section{BIBLIOGRAFÍA}

1. Bhamornsiri S. Wiggins C y Colson R. (2001). Comprehensive Income Disclosures. CPA Journal, 71 (10): pág. 54-57. Estados Unidos.

2. Consejo Mexicano de Normas de Información Financiera (2011). Norma Internacional de Información Financiera B-3 Estado de Resultado Integral. México.

3. Financial Accounting Standards Board FASB (1997). Statement of Financial Accounting Standards 130, Reporting Comprehensive Income. Estados Unidos.

4. International Accounting Standards Board IASB (2010). El Marco Conceptual para la Información Financiera. Inglaterra.

5. International Accounting Standards Board IASB (2012). NIC 1 Presentación de Estados Financieros. Inglaterra.

6. Jara, Luis y Abarca Allyson (2009). Primera Aplicación en Chile del Estado de resultados Integrales. Una aproximación a sus principales componentes y características. Artículo publicado en la revista CAPIC Review Vol. 7. Chile.

7. Mattessich, R. (2002). Teoría del Excedente Limpio y su Evolución: Revisión y Perspectivas Recientes, Energiea: Revista Internacional de Filosofía y Epistemología, 1 (2): pág.49.79, 
Universidad de Ciencias Empresariales y Sociales de Buenos Aires, Argentina.

8. Maines, L. y Mcdaniel, L. (2000). Effects of Comprehensive Income Characteristics on Nonprofessional Investors Judgments: The Role of Financial Statement Presentation Format. Accounting Review, 75 (2): pág. 179-208. Estados Unidos.

9. Sousa, F. Francisco (2009). Fundamentos Conceptuales del Resultado Global.Revista de Con-
tabilidad-Spanish Accounting Review Vol. 12 - No 2,pág. 215-252.España.

10. Superintendencia del Mercado de Valores (2011). Información financiera de empresas cotizantes en el mercado bursátil, disponible en la web www.smb.gob.pe. Perú

11. Vilchez, Percy. (2004). Armonización de Normas Contables en un mundo global. Análisis y Perspectivas. Trabajo Nacional para el Congreso Nacional de Contadores Públicos. Perú. 